\title{
Axis controlled movement of robot using brushless DC motor drive Deepak Batra ${ }^{1}$, Sanjay Sharma ${ }^{2}$ and Rajeev Ratan ${ }^{3}$ \\ ${ }^{1}$ ECE Deptt., C.I.T.M. Faridabad, Haryana; ${ }^{2}$ Thapar University, Patiala, Punjab, India \\ ${ }^{3} E \&$ IE Deptt., Apeejay College of Engg., Sohna, Gurgaon, Haryana, India \\ sanjay_eced@rediffmail.com
}

Abstract: This work attempts to study the simulation of the kinematics routine for the axis control of the robot using brushless DC motor drive. The main motivation is to perform kinematics routines as well as control the robot in real-time. The graphical user interfaces (GUI) has been developed in Matlab. The GUI has been designed such that the user can get a feel for the kinematics of the 5-axis articulated robot. The system was developed for demonstrating the basic trajectory planning method for the axis control of robot. Further the trajectory planning platform was tested for a particular experiment and was found to be accurate enough for experimental purposes. This comprehensive software package allows the user to learn the various aspects of serial manipulator basics and also helps in exploring the concepts better by working in real-time.

Keywords: Brushless DC motors, MATLAB, SImulink, GUI, permanent magnet synchronous motors, robot Introduction

Robots are complex electromechanical systems where several electric drives are used to control the movement of articulated structures. The design of axis control systems for robots can be greatly facilitated by the Electric Drives library, which can model complete axes including motor drives, speed reducers, mechanical model of the arm, and controllers in the same diagram.

This case study presents the modeling and simulation of a sixdegrees-of-freedom robot manipulator using Electric Drives library blocks in combination with Simulink ${ }^{\circledR}$ blocks. The two main joints models are built using brushless DC motor drives that are connected to the rest of the manipulator through speed reducers (a model included in the Electric Drives library).

The control system, essentially of two position control loops, is built with Simulink blocks. The inner speed and torque control loops are already included in the drive model. The rest of the manipulator and its load are represented by two Simulink nonlinear models, one

for each motor drive. Detailed modeling is presented to demonstrate the versatility of the Electric Drives library. The operation of the joints using typical trajectories is simulated and results are presented.

Description of the Robot Manipulator

The robot considered in this example is a generalpurpose six-degrees-of-freedom robot manipulator (GMF S-360) of parallelogram linkage type. Six-Degrees-ofFreedom Robot Manipulator shows the structure of the robot and its workspace. The robot has six axes. The three axes $\left(\Theta_{1}, \Theta_{2}, \Theta_{3}\right)$ shown in the figure (Fig.1) are for arm positioning and the others $(\alpha, \beta, \gamma)$ are for orientation of the end effector. In the horizontal plane, the robot can cover a 300 degree arc $\left(\Theta_{1}=-150^{\circ}\right.$ to $\left.\Theta_{1}=150^{\circ}\right)$. The robot's axes are driven by brushless DC motors that are modeled by permanent-magnet synchronous motors fed by PWM inverters (AC6 drive model). Speed reducers of belt type and gearbox are used to transmit torque from the motors to the joints (Miller, 1989; Lamár, 2002).

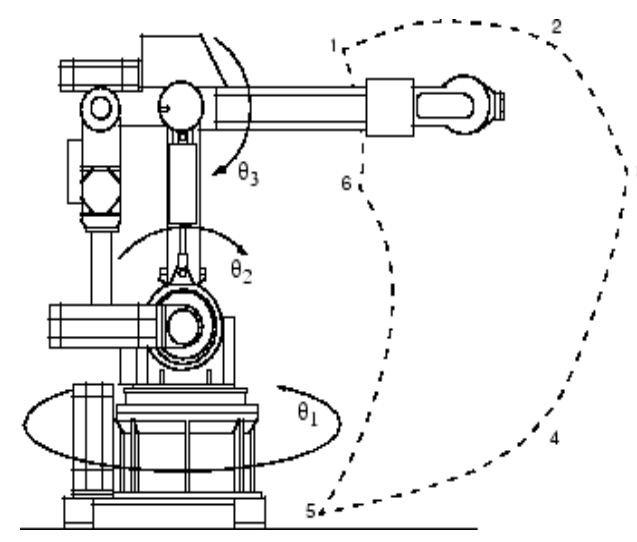

Fig. 1. Six-Degrees-of-freedom robot manipulator

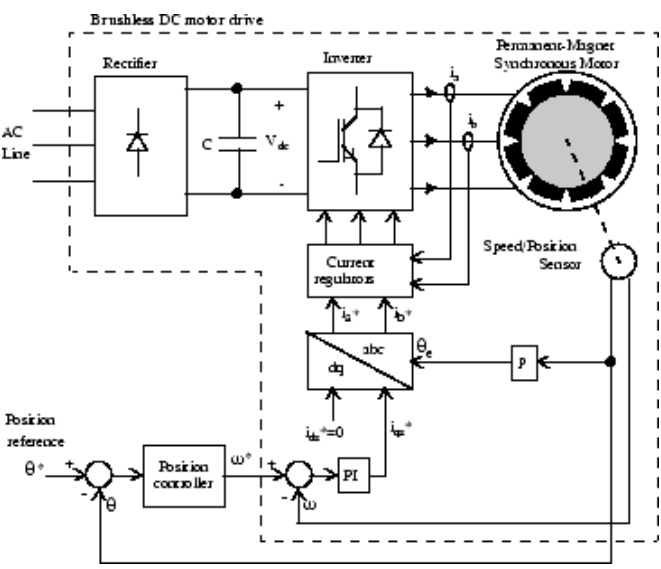

Fig. 2. Brushless DC motor drive for position control of robot joint

"Robot"

http://www.indjst.org
Position control systems for Joints 1 and 2 (Fig. 2)

We will consider in particular the two first joints, which drive the entire robot and its load. The first axis uses a $2 \mathrm{~kW}$ brushless DC motor and a 1:130 speed reducer. The second axis uses a $1 \mathrm{~kW}$ brushless DC motor and a 1:100 speed reducer. Brushless DC Motor Drive for Position Control of Robot Joint shows a simplified diagram of the position control system for one robot link (Schmidt et al., 2001).

The control system consists of three control loops connected in a cascade configuration: an outer position loop includes an inner speed control loop and an innermost current control loop. The PM synchronous motor is fed by a three-phase PWM inverter operating in currentcontrolled mode. Field-orientation scheme is used to decouple the variables so that flux and torque can be separately controlled by stator direct-axis current $i_{d s}$ and quadratureaxis current $\mathrm{i}_{\mathrm{qs}}$, respectively (Schmidt et al., 2000). The quadrature-axis current reference $\mathrm{i}_{\mathrm{qs}}{ }^{*}$ (which represents the torque command) is provided by the speed control loop. The direct-axis current reference $i_{d s}$ * Indian J.Sci.Technol. 
is kept equal to 0 . A speed/position sensor is used to provide the information required by the speed and position control loops. The rotor position is also required for coordinates conversion (dq to abc). Each motor drives the rest of the robot structure, including the other links and the load, through a speed reducer.

\section{Methodology}

Modeling the robot position control systems

The entire drive system for the robot's two first joints, including motor drives, speed reducers, equivalent loads, and controllers can be modeled in the same diagram using blocks from the electric drives library and Simulink

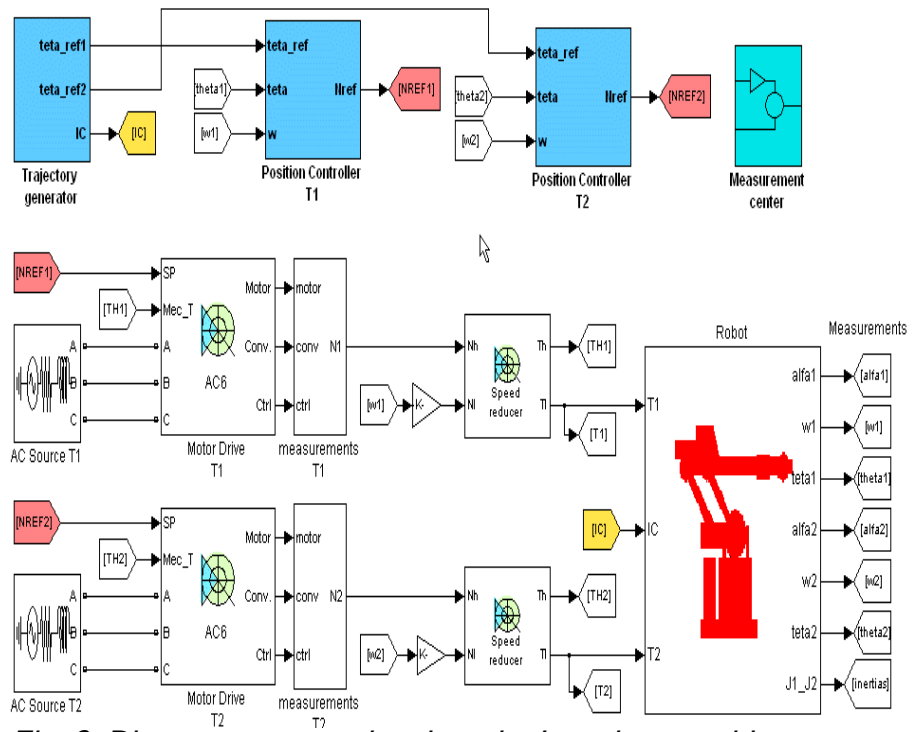

Fig. 3. Diagram representing the robot's main axes drive systems

libraries, as shown in Fig. 3 .

The brushless DC motor drives are represented by two AC6 (PM synchronous motor drive) blocks from the Electric Drives library. This block models a complete brushless DC motor drive including a permanent-magnet synchronous motor (PMSM), an IGBT inverter, speed controller, and current controller (Spong \& Vidyasagar, 1989). The AC6 inputs are the speed commands and the outputs are the motor speed, which are fed to the inputs of the speed reducers.

The speed reducers are modeled by two speed reducer blocks from the Electric Drives library. The inputs for these blocks are the motors' speeds, and the outputs are the torques from the low-speed sides, which are applied to the robot structure model. The speed reducers are characterized by their ratio and inertia and the stiffness and damping of input and output shafts (Korondi \& Hashimoto, 2003; Precup \& Preitl, 2004).

The speed reducers' output shafts are connected to the $T_{1}$ and $T_{2}$ inputs of a robot block that represents the rest of the robot structure. This block calculates the effective torque reflected to each joint. For each joint (numbered i), we can consider globally the other links effects as a single load reflecting to the joint a torque that is composed of three terms

$$
T_{L}=J_{i} \frac{d^{2} \theta_{i}}{d t^{2}}+C_{i} \frac{d \theta_{i}}{d t}+G_{i} \theta_{i}
$$

where $\theta_{i}$ is joint angular position, $J_{i}$ is inertia, $C_{i}$ is centrifugal and Coriolis coefficient, and $\mathrm{G}_{\mathrm{i}}$ is gravitational coefficient (Hunyár et al., 1998).

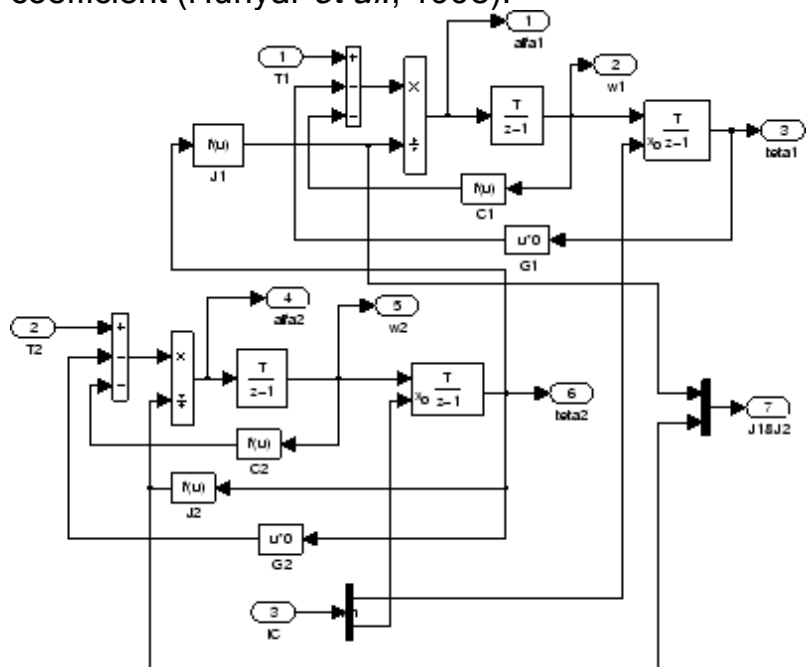

Fig.4. The robot model is built with Simulink blocks

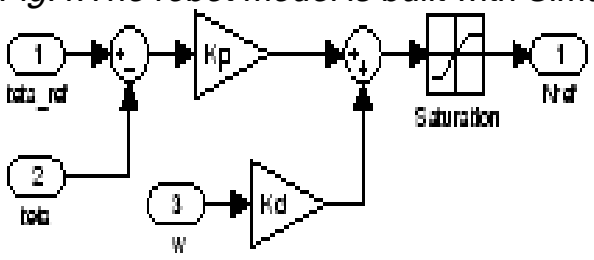

Fig. 5. Proportional \& derivative Simulink model

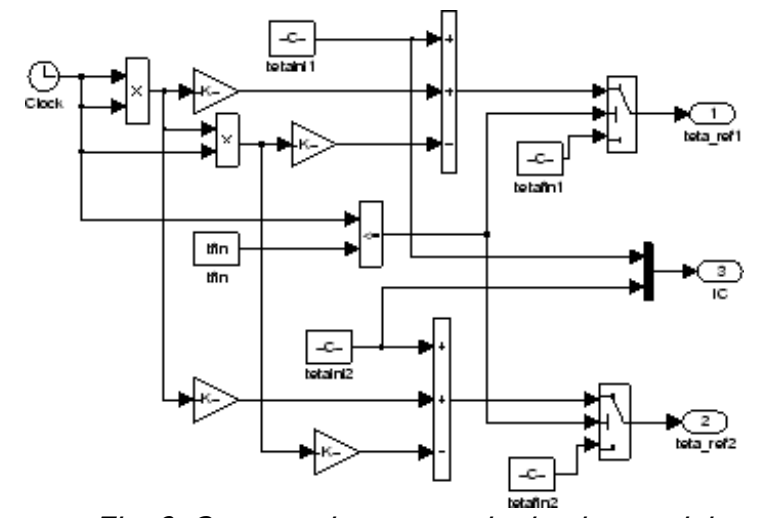

Fig. 6. Computed torque and adaptive model In

Fig.4, the parameters $\mathrm{J}_{1}, \mathrm{C}_{1}, \mathrm{G}_{1}, \mathrm{~J}_{2}, \mathrm{C}_{2}$, and $\mathrm{G}_{2}$ are functions of joint positions. Implement them by using polynomials or lookup tables.

The joint positions $\Theta_{1}$ and $\Theta_{2}$ are controlled by outer control loops that force $\Theta_{1}$ and $\Theta_{2}$ to follow the references imposed by the trajectories of the manipulator (Fig.5). Various algorithms can be used for these control loops. The most popular ones are proportional-derivative, computed torque, and adaptive (Fig.6). In this example, 


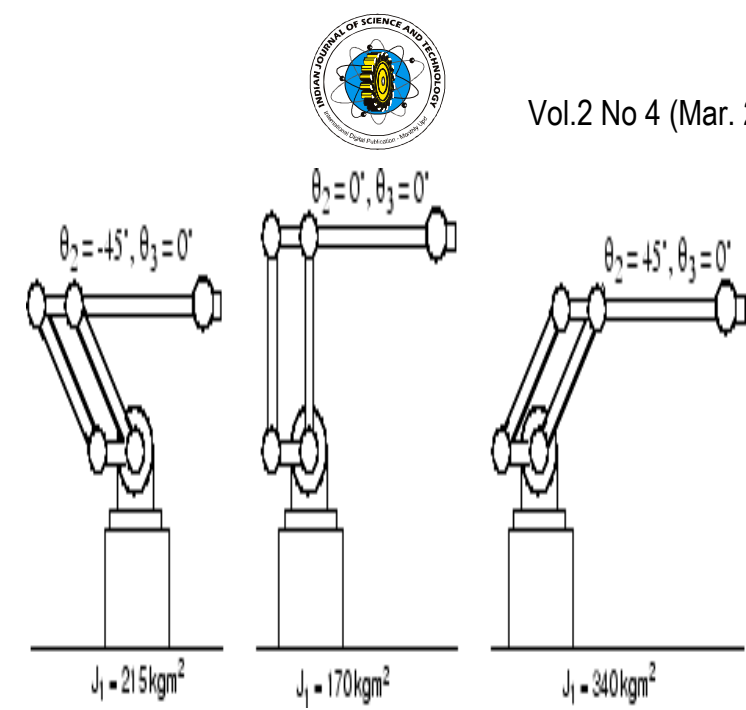

Fig. 7. Changes of robot structure during the programmed movement

proportional-derivative controllers are implemented for both axes (Yasuhiko \& Kinoshita, 1990).

Cubic polynomial test trajectories for robot motion are generated by the Trajectory Generator block.

The test trajectories consist of a movement from position 6 to position 3 in the workspace $\left(\Theta_{2}\right.$ varying from $-\pi / 4$ to $\Pi / 4$ ) while rotating around axis 1 from one position to another $\left(\Theta_{1}\right.$ varying from $-\Pi / 6$ to $n / 6)$. The parameters to be specified for this block are initial position $\left[\Theta_{1 \text { ini }}, \Theta_{2 \text { ini }}\right]$, final position $\left[\Theta_{1 \mathrm{fin}}, \Theta_{2 \mathrm{fin}}\right]$, and move time. Fig.7. shows the changes of robot structure during the programmed movement.

(Print users: vide cover page for colour plate)

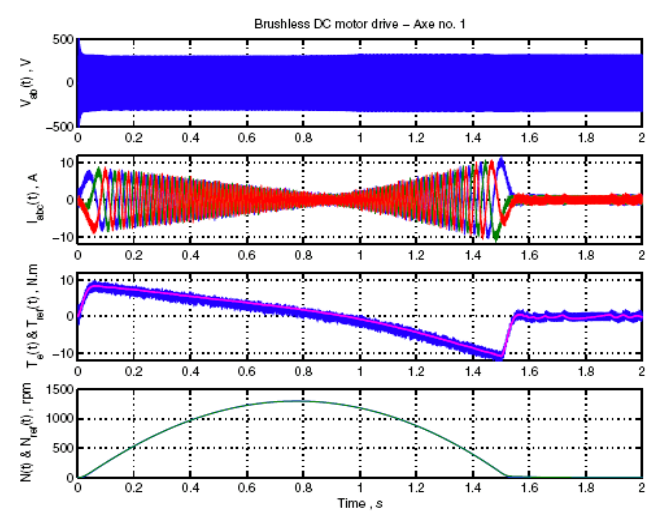

Fig. 9. Responses of the brushless DC motor drive of axis No. 1 during test trajectory

The variation of inertia due to structure changes is reflected to axis 1 as an inertia varying as a function of $\Theta_{2}$ (from $215 \mathrm{kgm}^{2}$ to $340 \mathrm{kgm}^{2}$ passing by a minimum of $\left.170 \mathrm{kgm}^{2}\right)$. The inertia reflected to axis 2 is a constant $\left(\mathrm{J}_{2}=50\right.$ $\left.\mathrm{kgm}^{2}\right)$. These inertia variations are represented by nonlinear functions implemented in the Robot block (Turmezei, 2002; Turmezei, 2003).

\section{Tracking Performance of the} Motor Drive

The test trajectories described above constitute one of the most demanding trajectories for the motor drive of the first and second joints. They are used here to evaluate the tracking performance of the two electric drive systems.

Research article

CIndian Society for Education and Environment (iSee)

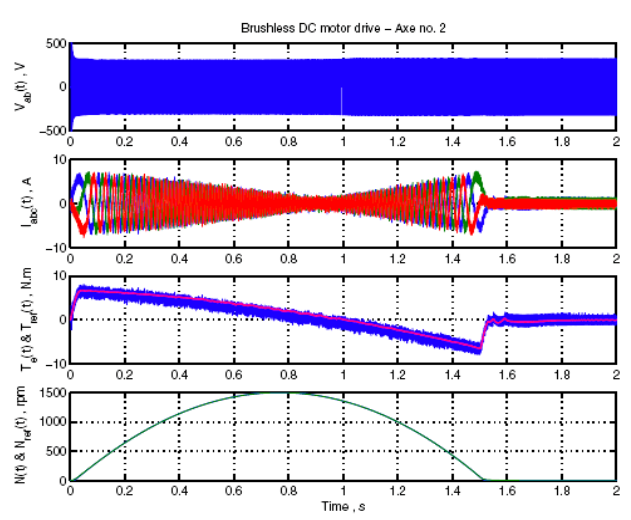

Fig. 10. Responses of the brushless DC motor drive of axis No. 2 during test trajectory

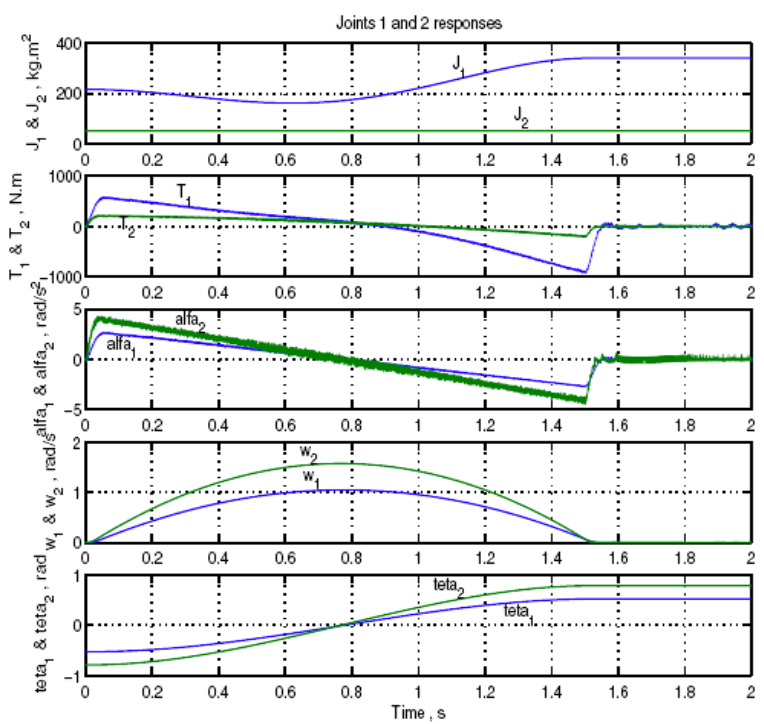

Fig. 8. Responses of the manipulator's joints 1 and 2 during a test trajectory

"Robot"

http://www.indjst.org
ISSN: 0974- 6846

In the simulation, the manipulator is programmed to rotate from $30^{\circ}$ to $30^{\circ}$ during 1.5 seconds, and at the same time the arm is moved from the back position $\left(\Theta_{2}=-45^{\circ}\right)$ to the most advanced position $\left(\Theta_{2}=45^{\circ}\right)$. The simulation is run using a time step of $1 \mu \mathrm{s}$.

The responses of the manipulator and the motor drives 1 and 2 are displayed on three scopes connected to the output variables of the AC6 and Robot blocks. The results are shown in Fig.8- 10.

\section{Results}

During the movement, the joint positions $\Theta_{1}$ and $\Theta_{2}$ follow the imposed cubic trajectories with low tracking error. The shapes of the speeds and accelerations are in agreement with theoretical predictions. The speed variations are second-degree curves and the accelerations are almost linear curves.

\section{Conclusion}

A large number of real-world data sets were collected from the experimental robot. In order to keep the robot around its operating point, but also for safety reasons, the data was collected using an experimental feedback control arrangement, which subsequently allows offline computations of the reference signals for the joint controllers.

The brushless DC motor drives behave very well during the test trajectories. The DC bus voltages are maintained at relatively constant levels during the deceleration of the motors. The developed torques are proportional to the motor currents' amplitudes. This demonstrates the good operation of the field-oriented control algorithms. As can be noted on the waveforms, the motor speeds track their reference profiles with very small errors.

The novel proposed

Deepak Patra et al. Indian J.Sci.Technol. 
method is easy to implement, and can be the basis of a cost-effective, highly reliable synchronous motor drives. Easy construction permanent magnet synchronous motors, simple low cost microcomputers (mainly DSPs) and integrated power modules can build up low and medium power, medium performance electrical drives, which can be used with for example electrical vehicle (wheel-chairs for disabled people, electrical mopeds, golfcars, etc.).

\section{References}

1. Hunyár $M$, Kovács $K$, Németh $K$, Schmidt I and Veszprémi K (1998) Energy-Saving and NetworkFriendly Electrical Drives (in Hungarian), Műegyetemi Kiadó, Budapest. ISBN 9634205690.

2. Korondi $P$ and Hashimoto $H$ (2003) Intelligent space as an integrated intelligent system. In: Proc. of the $15^{\text {th }}$ Intnl. Conf. on Electrical Drives and Power Electronics EDPE. Podbanské (Vysoké Tatry). Slovakia, ISBN 808911445 4. pp: 24-31.

3. Lamár K (2002) DSP Control unit for a sensorless synchronous motor drive. Master's Thesis. Institute of Automation, Kandó Kálmán Faculty of Electrical Engineering,

4. Miller THE (1989) Brushless Permanent-Magnet and Reluctance Motor Drives, Clarendon Press, Oxford.

5. Precup RE and Preitl S (2004) Fuzzy logic decision rules in two input single output linear timevarying systems control. In: Proc. Symposium on Applied Computational Intelligence SACl 2004, ISBN 963715425 4. pp: 69-78.

6. Schmidt I, Vincze K and Veszprémi K (2000) Servo and Robot Drives. Müegyetemi Kiadó, Budapest.

7. Schmidt I, Vincze K, Veszprémi $K$ and Seller B (2001) Novel PWM current vector control method of the PM synchronous servo drive with rectangular field. EPE-PEMC. Kosice. 7, 75-80.

8. Spong MW and Vidyasagar M (1989) Robot dynamics and control. John Wiley \& Sons, New York.

9. Turmezei P (2002) Einige Methoden der Erhöhung des Wirkungsgrades von Solarzellen, Kandó Conference 2002, Budapest, ISBN 9637158030.

10. Turmezei $P$ (2003) A few aspects on solarcellbased energy-supply of mechatronic-units (in Hunagrian), $3^{\text {rd }}$ Intnl. Symposium on Mechatronics, Budapest, ISBN 9637154221.

11. Yasuhiko D and Kinoshita S (1990) Brushless servomotors fundamentals and applications, Clarendon Press, Oxford. 